\title{
Zeroing Dynamics Method for Motion Control of Industrial Upper-limb Exoskeleton System with Minimal Potential Energy Modulation
}

\begin{abstract}
Accurate motion control of industrial upper-limb exoskeleton can provide efficient assistance for subjects to perform various industrial manipulation tasks. In most motion control scenarios of upper-limb exoskeletons, the variations of potential energy frequently reach to a high level of oscillations, leading to the reconstructed motion uncomfortable or dangerous. In this paper, in order to achieve minimal potential energy variation and accurate motion control of the upper-limb exoskeleton, we propose a novel motion planning strategy with minimal potential energy modulation. Such motion resolution scheme is formulated as an optimization problem and solved by the zeroing dynamics (ZD) to achieve elegant global convergence. Simulation and experiment results show that the potential energy variation range of the upper-limb exoskeleton can be significantly decreased by average $99.34 \%$ in both $X-Y$ and $X-Z$ planes, in addition to finishing tracking the desired motion path accurately. All of these demonstrate that the efficiency and superiority of the proposed method for potential energy minimization during achieving accurate motion planning and control.
\end{abstract}

Keywords: Exoskeleton, upper limb, kinematic control, zeroing neural network, potential energy 


\section{Introduction}

Nowadays, As a new type of service robots, exoskeletons have appeared to help humans to facilitate their mobility $[1,2]$. Industrial upper-limb exoskeletons are a mainstream type of exoskeletons which are primitively designed for upper-limb motion assistance, by following physiological modality and motion characteristics of upper extremities [3]. Research and development of upperlimb exoskeleton systems have received a lot of attentions recently, and many methods have been proposed to improve the functionality in different aspects for upper-limb exoskeletons [4]. For example, redundancy issues of humanexoskeleton systems for motion control have been investigated by analyzing forward and inverse kinematics $[5,6]$.

The motion of lower/upper extremities from humans is very dextrous because of inherently-natural redundancy which can be reflected by some limited kind patterns of synergy in actuation level $[7,8]$. As the number of degreesof-freedom (DOFs) of joints/muscles is much more than that of the desired motion dimensions, accurate motion control of upper limbs with redundancy by exoskeletons is essential and necessary. Efficient redundant motion control of upper-limb exoskeletons may be challenging because extra DOFs for resolution is needed and optimization of performance indexes should be involved [9]. Under planned/desired path for the end-effector/end-point, a upper-limb exoskeleton's joint angle can be conventionally resolved through generalized inverse of its Jacobian matrix directly $[10,11]$. Such way of processing might suffer from the drawback of unacceptable computational overload in real-time implementation and neglects required performance indexes (e.g., joint limits and mechanical energy consumption), so as to guarantee a reasonable motion planning. Such time-intensive tasks can be promising handled by neural networks which possess parallel processing ability $[12,13]$. Recurrent neural networks become an excellent alternative for inverse kinematic redundancy resolution of arm-type and hand-type robots $[14,15]$, exhibiting strong convergence ability and adaptivity to time-variant external factors [16]. Recently, as a powerful alternative solver 
for nonlinear/linear time-varying and static problems, zeroing neural network (ZNN) or its variant called zeroing dynamics (ZD) method have been proposed and extended to solve robot motion planning issues [17], providing an efficient way to deal with the defined error to achieve a good tracking capability.

On the other hand, in addition to effectuating accurate motion control, the potential energy of the exoskeleton systems is an important index for maintaining reliable planning and control performance [18], and maintaining the potential energy at a desired level is beneficial to support body weight by gravity compensation $[19,20]$. When fulfilling planned paths using upper-limb exoskeleton, decreasing potential energy variations during the same motion control tasks reflects less oscillations of the center of mass, which can avoid unexpected vibration caused by gravity and enhance system safety. However, currently there is very little work on potential energy minimization issue for redundancy motion control of upper-limb exoskeletons. Existing methods may not utilize the potential energy of the system as an additional index involved in motion planning tasks. Motivated by these points, inspired by incorporating the elegant solution ability of ZD into inverse kinematics resolution, this paper aims to make breakthroughs by proposing a novel motion control scheme for the upper-limb exoskeleton system which can minimize its potential energy to significantly decrease its variations during motion control process. The contributions of this work are summarized as follows:

1) To the best of our knowledge, this work may be the first work to propose a ZD-based inverse kinematics resolution scheme with minimal potential energy modulation for motion control of upper-limb exoskeleton systems.

2) The motion planning with potential energy minimization of the upperlimb exoskeleton is comprehensively formulated to a new optimization criteria by equipping a new constraint on potential energy, and the resultant ZD model is proposed and exploited to guarantee the global convergence of the redundancy resolution with minimal potential energy modulation.

3) Both simulation and experiment results demonstrate the efficiency of the proposed redundancy resolution for the upper-limb exoskeleton system. Seen 

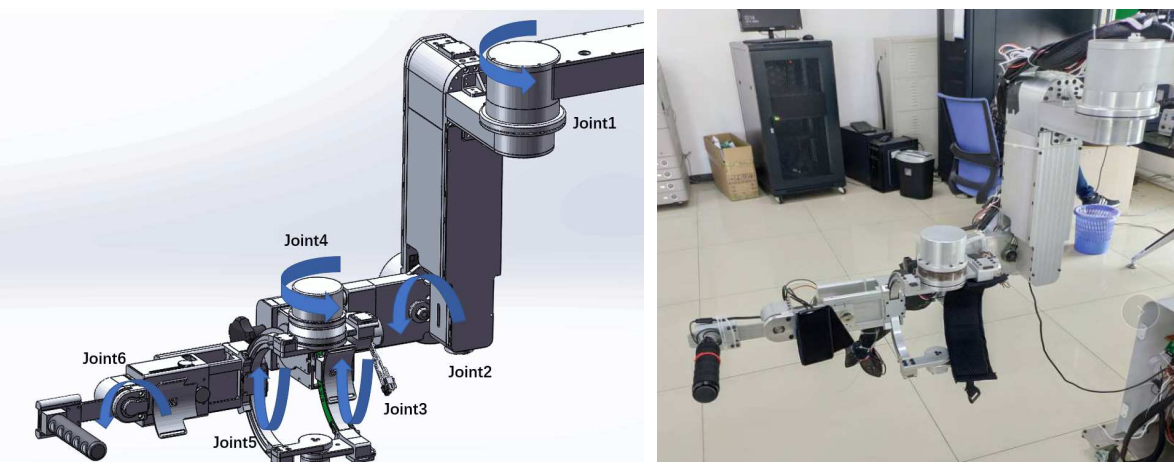

Figure 1: The upper-limb exoskeleton robot system. Left: the designed prototype; right: the developed system.

from the simulations and the experiments, the desired accurate motion control can be achieved. At the same time, the proposed potential energy minimization scheme makes the variation levels of the potential energy of the upper-limb exoskeleton system significantly lowered during motion control.

\section{Problem Formulation}

\subsection{Kinematics Modeling of Upper-limb Exoskeleton System}

The industrial upper-limb exoskeleton system is shown in Fig. 1. It possesses six links and six revolute joints. The masses of the links mainly focus on their proximal parts since the motors and the gears are equipped concentratively close to joints. The D-H parameters of the upper-limb exoskeleton system is summarized in Table 1. According to the mechanism of the upper-limb exoskeleton system in Fig. 1, its total potential energy is

$$
\begin{aligned}
U= & -m_{1} g L c_{1} \sin \theta_{2}+m_{2} g\left(\cos \theta_{2} \sin \theta_{3} \sin \theta_{4} L c_{2}\right. \\
& \left.-\sin \theta_{2} \cos \theta_{4} L c_{2}-L_{1} \sin \theta_{2}\right)-m_{3} g\left[L c_{3} \sin \theta_{6}\right. \\
& \left(\cos \theta_{2} \sin \theta_{3} \cos \theta_{4} \sin \theta_{5}+\sin \theta_{2} \sin \theta_{4} \sin \theta_{5}\right. \\
& \left.-\cos \theta_{2} \cos \theta_{3} \cos \theta_{5}\right)+L c_{3} \cos \theta_{6} \cos \theta_{2} \sin \theta_{3} \sin \theta_{4} \\
& -\sin \theta_{2} \cos \theta_{4}+\cos \theta_{2} \sin \theta_{3} \sin \theta_{4} L_{2} \\
& \left.-\sin \theta_{2} \cos \theta_{4} L_{2}-L_{1} \sin \theta_{2}\right]
\end{aligned}
$$


Table 1: D-H parameters of the upper-limb exoskeleton system.

\begin{tabular}{c|ccccc}
\hline Link & $a(\mathrm{~m})$ & $\alpha(\mathrm{rad})$ & $d(\mathrm{~m})$ & $\theta(\mathrm{rad})$ & Offset of $\theta(\mathrm{rad})$ \\
\hline 1 & 0 & $-\pi / 2$ & 0 & $\theta_{1}$ & 0 \\
2 & 0 & $-\pi / 2$ & 0 & $\theta_{2}$ & $-\pi / 2$ \\
3 & 0 & $-\pi / 2$ & 0.3109 & $\theta_{3}$ & $\pi / 2$ \\
4 & 0 & $\pi / 2$ & 0 & $\theta_{4}$ & 0 \\
5 & 0 & $\pi / 2$ & 0.2635 & $\theta_{5}$ & $\pi / 2$ \\
6 & 0.1 & 0 & 0 & $\theta_{6}$ & $\pi / 2$ \\
\hline
\end{tabular}

where $m_{1}, m_{2}$, and $m_{3}$ respectively denote the mass of link 1 , link 2 , and link $3, L_{1}, L_{2}$, and $L_{3}$ respectively denote the link length of link 1 , link 2 , and link 3 , and $L c_{1}, L c_{2}$, and $L c_{3}$ respectively denote the distance to the center of mass of link 1 , link 2 , and link 3 . The potential energy $U$ is evidently a function $U(\theta)$ on joint angles $\theta$ of the upper-limb exoskeleton system, and it changes as joint angle variable $\theta$ change with time $t$.

The modeling of the kinematics of the upper-limb exoskeleton system is according to the aforementioned D-H parameters in Table 1. As following the well-known kinematic chain's rule, the transformation matrix between base coordinate and end-effractor coordinate is depicted by

$$
T(\theta)=T\left(\theta_{1}\right) T\left(\theta_{2}\right) T\left(\theta_{3}\right) T\left(\theta_{4}\right) T\left(\theta_{5}\right) T\left(\theta_{6}\right)
$$

where the upper-limb exoskeleton's homogeneous transformation matrices are as follows

$$
\begin{gathered}
T\left(\theta_{1}\right)=\left[\begin{array}{cccc}
\cos \theta_{1} & 0 & -\sin \theta_{1} & 0 \\
\sin \theta_{1} & 0 & \cos \theta_{1} & 0 \\
0 & -1 & 0 & 0 \\
0 & 0 & 0 & 1
\end{array}\right] \\
T\left(\theta_{2}\right)=\left[\begin{array}{cccc}
\cos \left(\theta_{2}-\frac{\pi}{2}\right) & 0 & -\sin \left(\theta_{2}-\frac{\pi}{2}\right) & 0 \\
\sin \left(\theta_{2}-\frac{\pi}{2}\right) & 0 & \cos \left(\theta_{2}-\frac{\pi}{2}\right) & 0 \\
0 & -1 & 0 & 0 \\
0 & 0 & 0 & 1
\end{array}\right]
\end{gathered}
$$




$$
\begin{aligned}
& T\left(\theta_{3}\right)=\left[\begin{array}{cccc}
\cos \left(\theta_{3}-\frac{\pi}{2}\right) & 0 & -\sin \left(\theta_{3}-\frac{\pi}{2}\right) & 0 \\
\sin \left(\theta_{3}-\frac{\pi}{2}\right) & 0 & \cos \left(\theta_{3}-\frac{\pi}{2}\right) & 0 \\
0 & -1 & 0 & 0.3109 \\
0 & 0 & 0 & 1
\end{array}\right] \\
& T\left(\theta_{4}\right)=\left[\begin{array}{cccc}
\cos \theta_{4} & 0 & -\sin \theta_{4} & 0 \\
\sin \theta_{4} & 0 & \cos \theta_{4} & 0 \\
0 & 1 & 0 & 0 \\
0 & 0 & 0 & 1
\end{array}\right] \\
& T\left(\theta_{5}\right)=\left[\begin{array}{cccc}
\cos \left(\theta_{5}+\frac{\pi}{2}\right) & 0 & \sin \left(\theta_{5}+\frac{\pi}{2}\right) & 0 \\
\sin \left(\theta_{5}+\frac{\pi}{2}\right) & 0 & -\cos \left(\theta_{5}+\frac{\pi}{2}\right) & 0 \\
0 & 1 & 0 & 0.2635 \\
0 & 0 & 0 & 1
\end{array}\right] \\
& T\left(\theta_{6}\right)=\left[\begin{array}{cccc}
\cos \left(\theta_{6}+\frac{\pi}{2}\right) & -\sin \left(\theta_{6}+\frac{\pi}{2}\right) & 0 & 0.1 \cos \left(\theta_{6}+\frac{\pi}{2}\right) \\
\sin \left(\theta_{6}+\frac{\pi}{2}\right) & \cos \left(\theta_{6}+\frac{\pi}{2}\right) & 0 & 0.1 \sin \left(\theta_{6}+\frac{\pi}{2}\right) \\
0 & 0 & 1 & 0 \\
0 & 0 & 0 & 1
\end{array}\right]
\end{aligned}
$$

Equation (1) addresses the kinematics characteristics of the developed upperlimb exoskeleton system, and it reflects a nonlinear coupled mapping betweeen joint angular variable $\theta$ and position/oritention of the end-effector/end-point. Since all the joints of the upper-limb exoskeleton system are revolute joints and control of the orientation of the end-effector/end-point is not considered in this work, attitude control is not necessary for positioning of the end-effector/endpoint in motion planning.

For its joint angle variable $\theta(t)=\left[\theta_{1}(t), \theta_{2}(t), \cdots, \theta_{6}(t)\right] \in R^{6}$, and its Cartesian coordinate $r(t) \in R^{3}$ in the workspace can be described as the following nonlinear relation from (1):

$$
r(t)=f(\theta(t))
$$

where mapping function $f(\cdot)$ reflects geometrical and mechanistic information of the upper-limb exoskeleton system. Such mapping from the exoskeleton joint 
space to a its manipulation workspace at the position level and is highly-coupled nonlinear. The mapping at the velocity level can be simplified by performing derivations on both sides of $r(t)=f(\theta(t))$ with respect to time as follows

$$
\dot{r}(t)=J \dot{\theta}(t)
$$

where $J \in R^{3 \times 6}$ denotes the Jacobian matrix which is generated by $J(\theta(t))=$ $\partial f / \partial \theta$. It is worth noting here that, involvement of dynamics for optimal control may require solution of Hamilton-Jacobi (HJ) inequality and the HamiltonJacobi-Bellman (HJB) equation, but finding explicit closed-form solutions for HJ inequality and HJB equation might be hard or impossible in practice.

\subsection{Minimal Modulation of Potential Energy}

Upper-limb exoskeletons have to avoid enduring large-scale variation of potential energy during motion control process. Especially, in some industrial scenarios [21], the manipulation workspace should be limited due to the narrow task space, the joint angle magnitudes should be suitably restricted. If potential energy of such upper-limb exoskeletons changes at a quite large range vertically, it may make the subjects uncomfortable due to unexpected physical interaction. Thus, design of motion planning strategy for upper-limb exoskeletons should take into account enhancing weight-loss effect by limiting the bounds of potential energy variations. In this paper, we propose a potential energy minimal modulation scheme for the motion planning and control of the upper-limb exoskeleton. To exploit optimal solutions with potential energy minimization solved, the following constrained objective is proposed to be minimized:

$$
\begin{aligned}
\operatorname{minimize} & \frac{d U}{d t} \\
\text { subject to } & J \dot{\theta}=\dot{r}_{d}
\end{aligned}
$$

The optimization formulation (3) aims to make minimization of the time derivative of potential energy of the upper-limb exoskeleton system during motion planning with desired path tracked. To evaluate the quality of variation conditions for potential energy of the upper-limb exoskeleton system, we propose to 
use the following potential energy variation index (PEVI)

$$
\mathrm{PEVI}=\left|\int_{0}^{T} U-\max (U) d t\right|
$$

If PEVI is larger, it means that the upper-limb exoskeleton has a worse potential energy minimization effect, otherwise, it has better potential energy minimization effect.

\section{Zeroing Dynamics (ZD) Method for Minimally Modulating of Po- tential Energy}

In order to perform minimal modulation of potential energy of the upperlimb exoskeleton, in this section, we propose a ZD model to solve (3) problem. Firstly, the following error processing function is defined to tracking the desired position of the end-effector/end-point and the variation of the potential energy

$$
e=\left[\begin{array}{c}
f(\theta)-r_{d} \\
U-U\left(\theta_{0}\right)
\end{array}\right] \in R^{4}
$$

where $U\left(\theta_{0}\right)$ denotes the potential energy of the upper-limb exoskeleton at the initial joint angle $\theta(0)$. Based on the ZD design principle, we handle the error function $e$ by exploiting the following first-order differential equation

$$
\dot{e}=-\Lambda \Phi(e)
$$

where $\Phi(\cdot): R^{4 \times 4} \rightarrow R^{4 \times 4}$ can be an array of odd monotonically-increasing functions $\phi(\cdot)$, and parameter matrix $\Lambda$ is a diagonal matrix

$$
\Lambda=\left[\begin{array}{cccc}
\gamma & & & \\
& \gamma & & \\
& & \gamma & \\
& & & \gamma
\end{array}\right] \in R^{4 \times 4}
$$

with constant scalar $\gamma>0$. According to the error definition (5) and its processing method (6), one can have

$$
\left\{\begin{array}{l}
\frac{d f(\theta)}{d t}-\dot{r}_{d}=-\gamma \Phi_{1}\left(f(\theta)-r_{d}\right) \\
\dot{U}=-\gamma \Phi_{2}\left(U-U\left(\theta_{0}\right)\right)
\end{array}\right.
$$


where $\Phi_{1}(\cdot): R^{3 \times 3} \rightarrow R^{3 \times 3}$ and $\Phi_{2}(\cdot): R \rightarrow R$ respectively denote the subarray of $\Phi(\cdot)$. As $J \dot{\theta}=\frac{d f(\theta)}{d t}$ and $\dot{U}=\frac{\partial U}{\partial \theta} \dot{\theta}$ always hold, the above equations further become

$$
\left\{\begin{array}{l}
J \dot{\theta}=-\gamma \Phi_{1}\left(r-r_{d}\right)+\dot{r}_{d} \\
\frac{\partial U}{\partial \theta} \dot{\theta}=-\gamma \Phi_{2}\left(U-U\left(\theta_{0}\right)\right)
\end{array}\right.
$$

Specifically, if linear activation functions $\phi\left(e_{i}\right)=e_{i}$ are used, the above equations become

$$
\left\{\begin{array}{c}
J \dot{\theta}=-\gamma\left(r-r_{d}\right)+\dot{r}_{d} \\
\frac{\partial U}{\partial \theta} \dot{\theta}=-\gamma\left(U-U\left(\theta_{0}\right)\right)
\end{array}\right.
$$

In order to develop the $\mathrm{ZD}$ solver for minimal potential energy modulation for the motion control, by letting $z=\theta$ we reformulate equations (7) to the following linear equation

$$
A \dot{z}=b
$$

where the coefficients are

$$
A=\left[\begin{array}{c}
J \\
\frac{\partial U}{\partial \theta}
\end{array}\right] \in R^{4 \times 6} \text { and } b=\left[\begin{array}{c}
-\gamma \Phi_{1}\left(r-r_{d}\right)+\dot{r}_{d} \\
-\gamma \Phi_{2}\left(U-U\left(\theta_{0}\right)\right)
\end{array}\right] \in R^{4}
$$

To solve linear equations $A \dot{z}=b$ by the ZD method, we reformulate it to obtain

$$
A^{T} A \dot{z}=A^{T} b
$$

Thus, we have the following ZD model for solving motion planning with minimal modulation of potential energy for the upper-limb exoskeleton

$$
W \dot{z}=v
$$

where $W=A^{T} A$ and $v=A^{T} b$. The ZD model (8) is able to make the upperlimb exoskeleton track the desired path $r_{d}$ and its potential energy converge to the initial energy point $U\left(\theta_{0}\right)$. To demonstrate this theoretically, firstly we show some theoretical results by giving three theorems as follows.

Theorem 1. For motion resolution and minimization of potential energy for the upper-limb exoskeleton by ZD model (8), if monotonically-increasing odd activation functions are used, the state variable $z$ of (8) can globally converge to the desired theoretical solution $z^{*}$. 
Proof. In order to further show its convergence property, we define the following Lyapunov function for analyzing ZD model (8) as follows

$$
V=\frac{1}{2}\|e\|_{2}^{2}=\frac{1}{2} \sum_{i=1}^{4} e_{i}^{2} \geq 0
$$

where $\|\cdot\|_{2}$ denotes the two norm of vector. Correspondingly, the time derivative of $V$ is

$$
\dot{V}=\sum_{i=1}^{4} e_{i} \dot{e}_{i}=\sum_{i=1}^{4}-\gamma e_{i} \phi\left(e_{i}\right)
$$

As $\phi(\cdot)$ is an odd monotonically-increasing function, it will satisfy [22]

$$
e_{i} \phi\left(e_{i}\right) \begin{cases}>0, & e_{i} \neq 0 \\ =0, & e_{i}=0\end{cases}
$$

Therefore, the time derivative of Lyapunov function $\dot{V}$ is negative definite. It means that the error $e_{i}$ can globally convergence to zero, which indicates that the desired theoretical solution $z^{*}$ can be achieved by $z$. The proof is complete.

Theorem 2. For motion resolution and minimization of potential energy for the upper-limb exoskeleton by ZD model (8), if the linear activation function $\phi\left(e_{i}\right)=e_{i}$ is used for (8), the state variable $z$ can globally exponentially converge to the desired theoretical solution $z^{*}$.

Proof. Recall the defined Lyapunov function

$$
V=\frac{1}{2} \sum_{i=1}^{4} e_{i}^{2}
$$

and its negative-definite time-derivative function $\dot{V}$

$$
\dot{V}=\sum_{i=1}^{4} \dot{e}_{i} e_{i}=\sum_{i=1}^{4}-\gamma \phi\left(e_{i}\right) e_{i}
$$

When the linear activation function is used, i.e., $\phi\left(e_{i}\right)=e_{i}$, then the timederivative function becomes

$$
\dot{V}=\sum_{i=1}^{4}-\gamma e_{i}^{2} \leq 0
$$


As $\dot{e}_{i}=-\gamma e_{i}$, we can also have

$$
e_{i}=\exp (-\gamma t) e_{i}(0)
$$

Therefore the time derivative $\dot{V}$ of Lyapunov function $V$ becomes

$$
\dot{V}=\sum_{i=1}-\gamma \exp (-2 \gamma t) e_{i}^{2}(0) \leq 0
$$

It reveals that the vanishing of the Lyapunov function is exponentially stable and the solution process can exponentially converge. Thus $z$ can exponentially converge to the desired theoretical solution $z^{*}$. The proof is complete.

Theorem 3. For motion resolution and minimization of potential energy for the upper-limb exoskeleton by ZD model (8), if the following power-sigmoid activation function

$$
\phi\left(e_{i}\right)= \begin{cases}e_{i}^{p}, & \left|e_{i}\right|>1, p>1 \\ \frac{1-\exp \left(\xi e_{i}\right)}{1+\exp \left(\xi e_{i}\right)}, & \left|e_{i}\right|<1, \xi>1\end{cases}
$$

and the sine hyperbolic activation function

$$
\phi\left(e_{i}\right)=\frac{\exp \left(\xi e_{i}\right)-\exp \left(-\xi e_{i}\right)}{2}
$$

are used for the general ZD model (8), superior convergence can be achieved as compared with the case of using linear activation functions.

Proof. For the general ZD model (8), we define the same Lyapunov function

$$
V=\frac{1}{2} \sum_{i=1}^{4} e_{i}^{2}
$$

and examine its time derivative

$$
\dot{V}=\sum_{i=1}-\gamma \phi\left(e_{i}\right) e_{i} \leq 0
$$

According to the property of power-sigmoid functions [23], if the powersigmoid activation function is used, its time-derivative is

$$
\dot{V}= \begin{cases}-\gamma e_{i}^{p+1}<-\gamma e_{i}^{2}<0, & \left|e_{i}\right|>1 \\ -\gamma e_{i} \frac{1-\exp \left(\xi e_{i}\right)}{1+\exp \left(\xi e_{i}\right)} \leq-\gamma e_{i}^{2} \leq 0, & \left|e_{i}\right| \leq 1\end{cases}
$$




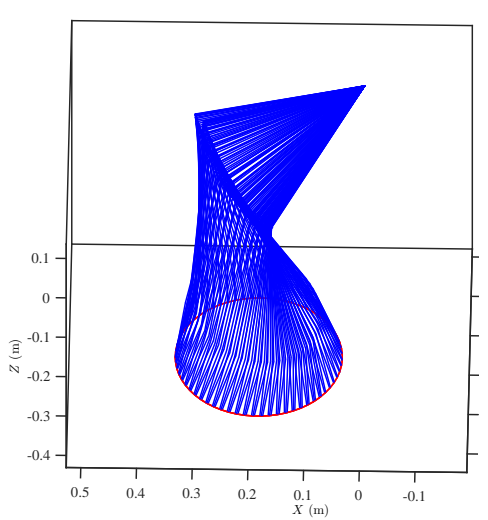

(a) Without potential energy minimization

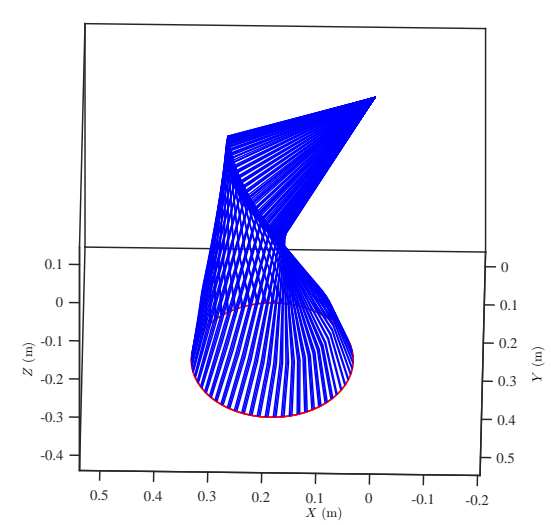

(b) With potential energy minimization

Figure 2: Comparison of circle path tracking performance in $X-Y$ plane for the upper-limb exoskeleton without and with potential energy minimization. The motion magnitudes of links and joints with potential energy minimization can be evidently reduced.

According to the property of sine hyperbolic functions [24], if the sine hyperbolic activation function is used, its time-derivative is

$$
\dot{V}=-\gamma e_{i} \frac{\exp \left(\xi e_{i}\right)-\exp \left(-\xi e_{i}\right)}{2} \leq-\gamma e_{i}^{2} \leq 0
$$

All of these imply that the vanishing rate of the Lyapunov function is larger than the case with linear activation functions. The proof is thus complete.

\section{Simulation Results}

In this section, we investigate the path tracking issue of the upper-limb exoskeleton model and evaluate its motion control performance by the aforementioned ZD model. The proposed potential energy minimization solution is integrated to reduce the potential energy variations. We compare the performance of the proposed potential-energy based solution with current solution without potential energy minimization involved. The simulation model of the upper-limb exoskeleton system is according to the D-H parameters in Table 1. The desired circular motion is planned in $X-Z$ and $X-Y$ planes with its radius being $0.10 \mathrm{~m}$. 


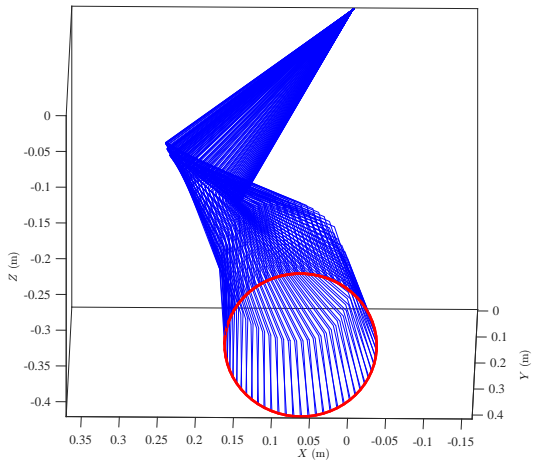

(a) Without potential energy minimization

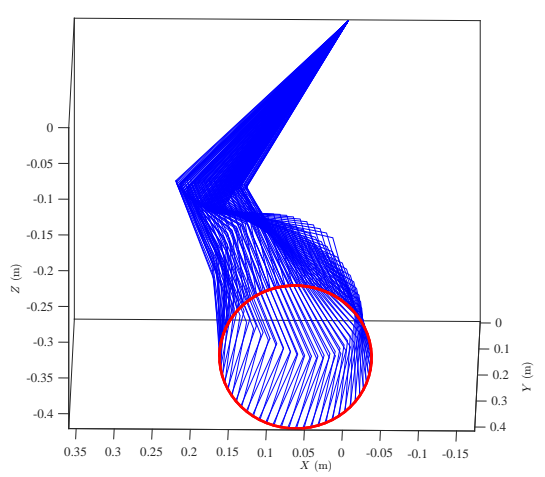

(b) With potential energy minimization

Figure 3: Comparison of circle path tracking performance in $X-Z$ plane for the upper-limb exoskeleton without and with potential energy minimization. The motion magnitudes of links and joints with potential energy minimization can be evidently reduced.

\subsection{Path Tracking}

We evaluate the motion control performance of the upper-limb exoskeleton based on the ZD method through examining the circle tracking in both $X-Y$ and $X-Z$ planes.

Firstly, the $X-Y$ plane's circle tracking performance of the upper-limb exoskeleton by the ZD is presented. Fig. 2, in which the piecewise straight lines in blue represent the body of the upper-limb exoskeleton, and the curves in red represent the trajectory of the end-point/effactor, shows the generated trajectory using the ZD without and with potential energy minimization starting from initial joint angle $\theta_{0}=[1.2 \pi / 8,1.2 \pi / 6,0,1.2 \pi / 3,-1.2 \pi / 6,1.2 \pi / 4]^{T}$. It can be obviously seen that the generated trajectories of the upper-limb exoskeleton without and with potential energy minimization successfully both track the desired circular path. Moreover, we could also observe from Fig. 2 that, when potential energy minimization is utilized for the motion-planning solution, the generated motions in the upper body part of the upper-limb exoskeleton are with less magnitudes, which may indicate that the variation range of the center of mass of the body of the upper-limb exoskeleton recedes. Next, circle path tracking in $X-Z$ plane for the upper-limb exoskeleton by ZD is shown by 

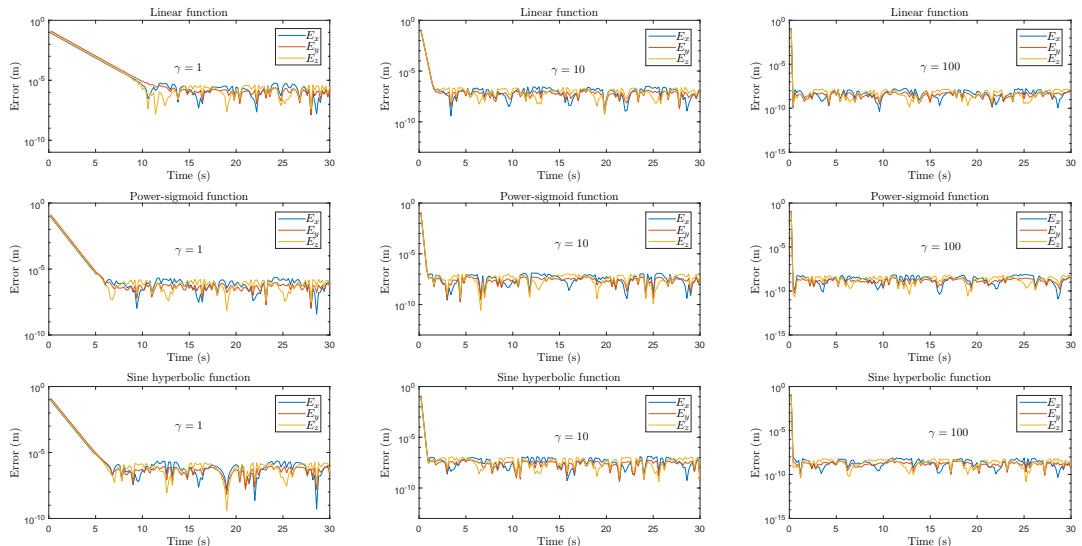

Figure 4: Position errors $\left[E_{x}, E_{y}, E_{z}\right]$ of the upper-limb exoskeleton for tracking the circle path in $X-Y$ plane. Different activation functions and different parameters $\gamma$ are used.

Fig. 2. The similar phenomena can also be observed in this case, that is, the variation range of the center of mass of the body of the upper-limb exoskeleton recedes as well. Fig. 6 and Fig. 7 respectively shows the joint angles without and with potential energy minimization in $X-Y$ and $X-Z$ planes. We can see that, in $X-Y$ plane, after potential energy minimal modulation is exerted, the magnitude of $\theta_{2}$ is obviously decreased, while in $X-Z$ plane, angles of most of the joints have been changed potential energy minimal modulation involved.

Fig. 4 and Fig. 5 show the position errors of the upper-limb exoskeleton for tracking the desired circle path by ZD with different types of activation functions (linear function, power-sigmoid function, and sine hyperbolic function) and parameters $\gamma$ in different scale. From these figures, we can see that the position errors are rather small in steady state. As $\gamma=100$ is configured, the position error level can be decreased to a very low level (around $10^{-10} \mathrm{~m}$ ). Additionally, faster convergence rate of the transient process can be achieved when power-sigmoid and sine hyperbolic activation functions are utilized for ZD models with the same parameter $\gamma$ as compared with the situation of using linear functions. All of these results validate that the inverse kinematics resolution based on ZD model for the upper-limb exoskeleton is efficient. 

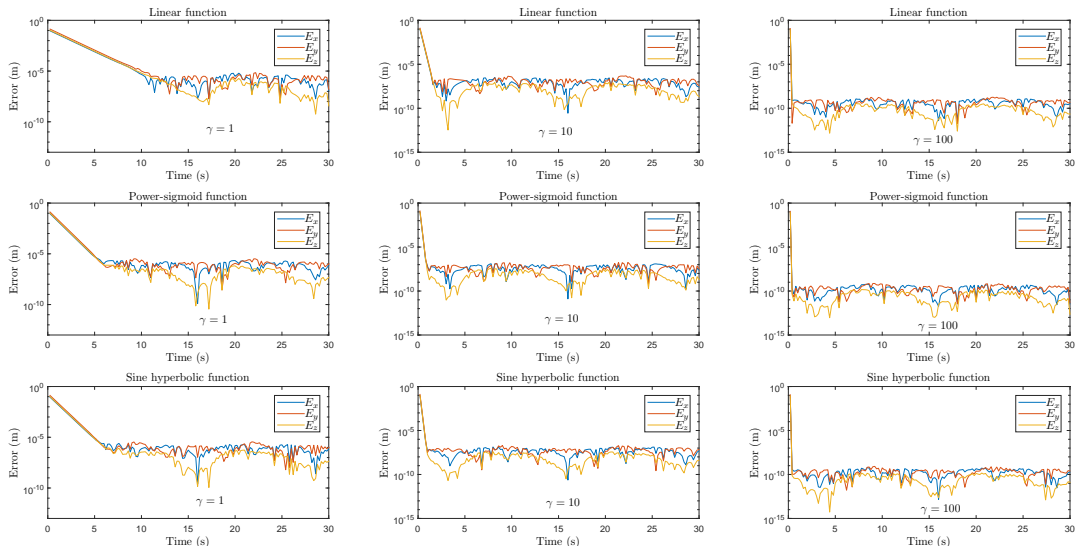

Figure 5: Position errors $\left[E_{x}, E_{y}, E_{z}\right]$ of the upper-limb exoskeleton for tracking the circle path in $X-Z$ plane. Different activation functions and different parameters $\gamma$ are used.

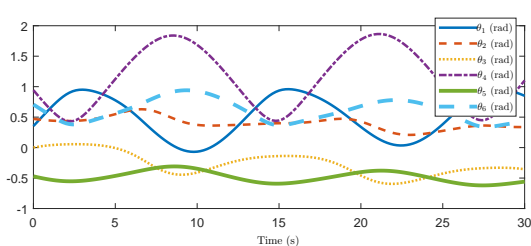

(a) Without potential energy minimization

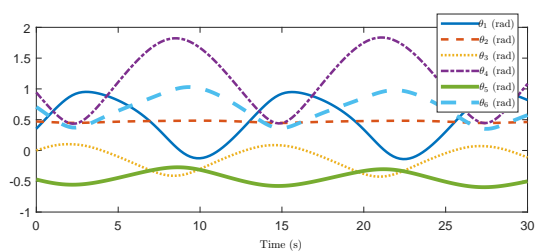

(b) With potential energy minimization

Figure 6: Comparison of joint angles $\theta$ in $X-Y$ plane for the upper-limb exoskeleton without and with potential energy minimization.

\subsection{Potential Energy Evaluation}

Fig. 8 and Fig. 9 further show the potential energy curves during motion control process for the upper-limb exoskeleton without and with minimization scheme adopted, starting from eight different initial joint angles. It can be evidently seen that, when the minimization scheme is used, the variation range of the potential energy is greatly reduced. When the minimization scheme by ZD with $\gamma=100$ is used, the potential energy is almost keeping at the same level of the initial energy state. Fig. 10 further shows the convergence performance of the potential energy of the upper-limb exoskeleton synthesized by ZD with different parameter $\gamma$, and we see that the convergence of potential energy can be accelerated when increasing $\gamma$ from 1 to 100. Table 2 shows the potential en- 


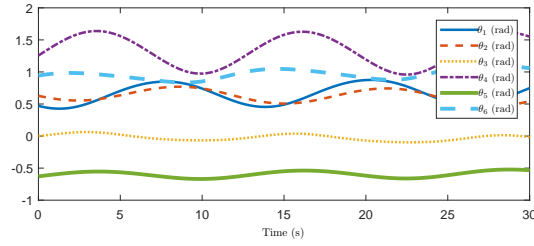

(a) Without potential energy minimization

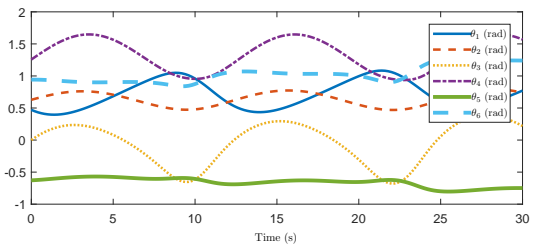

(b) With potential energy minimization

Figure 7: Comparison of joint angles $\theta$ in $X-Z$ plane for the upper-limb exoskeleton without and with potential energy minimization.

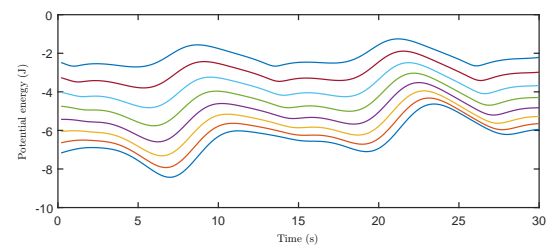

(a) Without potential energy minimization

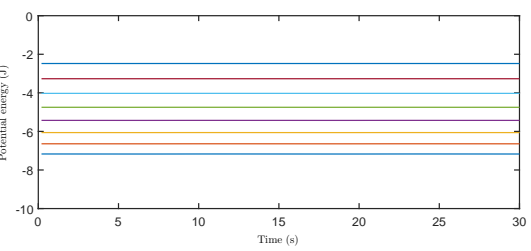

(b) With potential energy minimization

Figure 8: Potential energy variation comparison from the eight different initial joint angles in $X-Y$ plane, where $\gamma=100$ is used.

ergy variations before and after using the minimization scheme according to the proposed evaluation metric (4). In $X-Y$ plane, the average PEVI value without potential energy minimization is $44.63 \pm 9.69 \mathrm{~J} \cdot \mathrm{s}$, nevertheless, the average PEVI value with potential energy minimization is $0.02 \pm 0.01 \mathrm{~J} \cdot \mathrm{s}$. In $X$ - $Z$ plane, the average PEVI value without potential energy minimization is $83.22 \pm 7.68$ $\mathrm{J} \cdot \mathrm{s}$, and those with potential energy minimization is $0.06 \pm 0.01 \mathrm{~J} \cdot \mathrm{s}$. From such quantitative comparisons, we see that, the oscillation of the potential energy can be greatly suppressed after the proposed minimal modulation scheme used. All these results demonstrate that the potential energy minimization scheme based on ZD model for the upper-limb exoskeleton is efficient to diminish the potential energy as expected. In addition, the potential energy variations of the upper-limb exoskeleton are flattened from the different initial values of the potential energy by the proposed method, when the parameter $\gamma$ is increased, the variations become flat with faster convergence. During the whole motion 


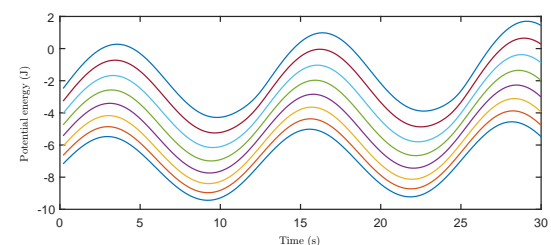

(a) Without potential energy minimization

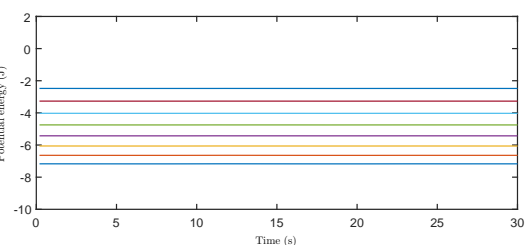

(b) With potential energy minimization

Figure 9: Potential energy variation comparison from the eight different initial joint angles in $X-Z$ plane, where $\gamma=100$ is used.
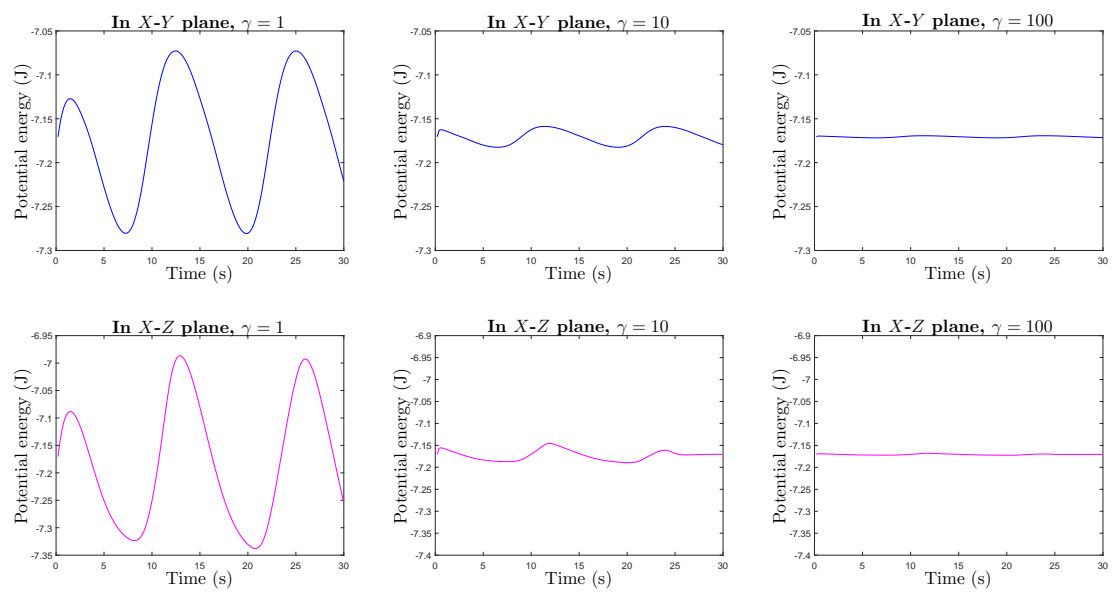

Figure 10: Potential energy variation comparisons with different $\gamma$ used from the same quintal joint angle of the upper-limb exoskeleton in $X-Y$ and $X-Z$ planes.

control process, the phenomena of minimal modulation on potential energy can appear based on the ZD-based method.

\section{Experiment Results}

The experiments are performed on the developed upper-limb exoskeleton system as shown in Fig. 1. The parameter $\gamma$ for the motion resolution is set as 100. In the six joints of the upper-limb exoskeleton system, six DC (direct current) brushless motors (Maxon, Switzerland) are equipped with attached drivers (Maxon-EPOS4, Switzerland). The servo control mode of the drivers is of position profile mode (PPM). The tracking paths are respectively set as the circular with diameter $0.2 \mathrm{~m}$ in $X-Z$ plane and $X-Y$ plane, and the reference 
Table 2: Average PEVI values $(\mathrm{J} \cdot \mathrm{s})$ of the upper-limb exoskeleton system of simulation from eight different initial joint angles.

\begin{tabular}{ccc}
\hline $\begin{array}{c}\text { Desired path plane } \\
\text { for motion control }\end{array}$ & $\begin{array}{c}\text { Without potential } \\
\text { energy minimization }\end{array}$ & $\begin{array}{c}\text { With potential } \\
\text { energy minimization }\end{array}$ \\
\hline$X-Y$ plane & $44.63 \pm 9.69$ & $\mathbf{0 . 0 2} \pm \mathbf{0 . 0 1}$ \\
$X-Z$ plane & $83.22 \pm 7.68$ & $\mathbf{0 . 0 6} \pm \mathbf{0 . 0 1}$ \\
\hline
\end{tabular}

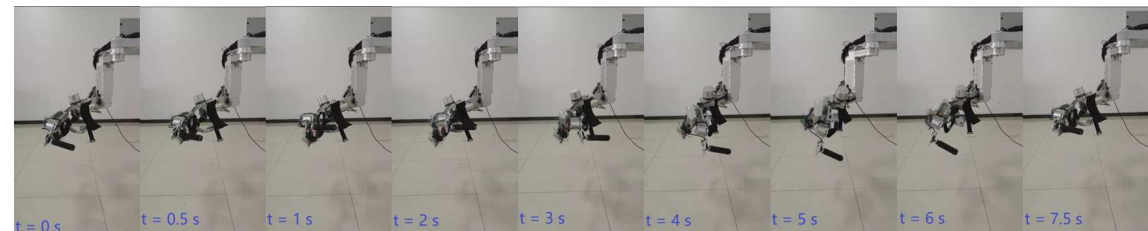

Figure 11: Snapshots of experiment process of the upper-limb exoskeleton system for tracking the desired path.

joint angles are computed according to the proposed motion control scheme with minimal potential energy modulation. For comparisons, the reference joint angles are also resolved without potential energy immunization for the same path target tracking. The joint angle data in the inverse resolution of the upper-limb exoskeleton system are interpolated and sent to the drivers with a renewal frequency being $20 \mathrm{~Hz}$. The timeslice of the experiment for the upperlimb exoskeleton system for doing the circle path tracking based on ZD models is shown as Fig. 11.

The path tracking experiments are performed in both $X-Y$ and $X-Z$ planes. In the tracking task in each plane, the potential energy minimization scheme is involved in the motion control module. Fig. 12 shows the path tracking performances in such two cases. In Fig. 12, we could observe that, with potential energy minimization utilized, the end-effector/end-point of the upper-limb exoskeleton system can keep tracking the desired circle path(s) in both $X-Y$ and $X-Z$ planes quite well.

Fig. 13 further shows the position errors of the end-effractor/end-point during path tracking tasks in both $X-Z$ and $X-Y$ planes with potential energy minimization, we can evidently see that the position errors in three dimensions $\left[E_{x}, E_{y}, E_{z}\right]$ are rather small which are lower than the level of $6 \mathrm{~mm}\left(<6 \times 10^{-3}\right.$ 


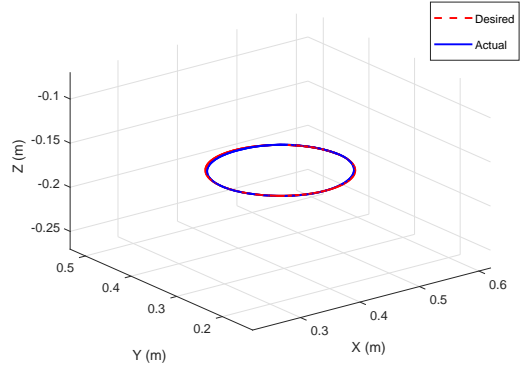

(a) Tracking performance in $X-Y$ plane

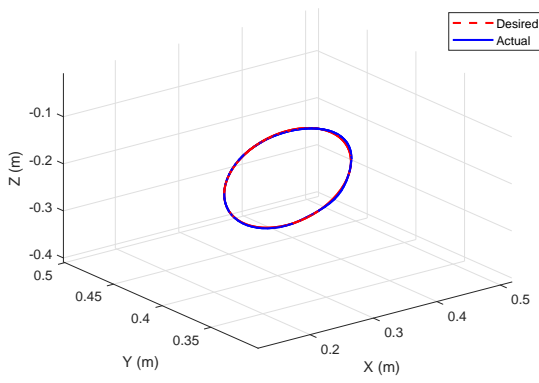

(b) Tracking performance in $X-Z$ plane

Figure 12: Experimental path tracking performance in $X-Y$ plane and $X-Z$ plane for the upper-limb exoskeleton system with minimal potential energy modulation.

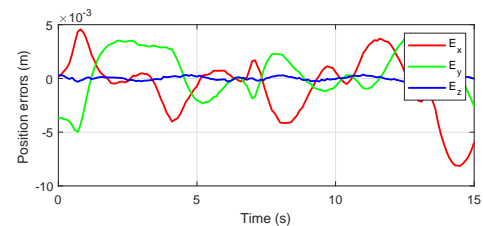

(a) Error performance in $X-Y$ plane

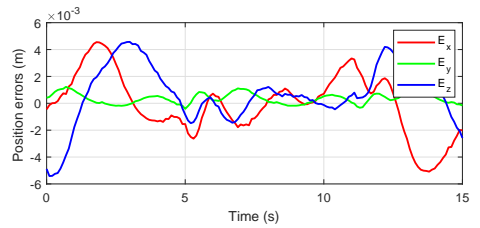

(b) Error performance in $X-Z$ plane

Figure 13: Experimental position tracking errors in $X-Y$ plane and $X-Z$ plane for the upperlimb exoskeleton system with potential energy minimized.

m). All of these experimental results can indicate that, after potential energy minimization is inducted, the motion control accuracy can still be guaranteed and the adopted ZD-based method is efficient in computing the referred joint angles for inverse kinematics resolution. Fig. 14 and Fig. 15 respectively shows the potential energy without and with its minimization scheme utilized in path tracking tasks in $X-Y$ and $X-Z$ planes. From the two figures, we evidently see that the oscillation of the potential energy is greatly eliminated when minimization scheme based on ZD is performed. Table 3 shows the comparisons of PEVI values according to (4) during the same motion control experiments of the upperlimb exoskeleton system, and we can see that the PEVI values are significantly decreased after potential energy minimization scheme used, i.e., from $3.816 \mathrm{~J} \cdot \mathrm{s}$ to $0.094 \mathrm{~J} \cdot \mathrm{s}$ in $X-Y$ plane and from $39.629 \mathrm{~J} \cdot \mathrm{s}$ to $0.243 \mathrm{~J} \cdot \mathrm{s}$ in $X-Z$ plane. The potential energy of the upper-limb exoskeleton system is decreased by $97.53 \%$ 


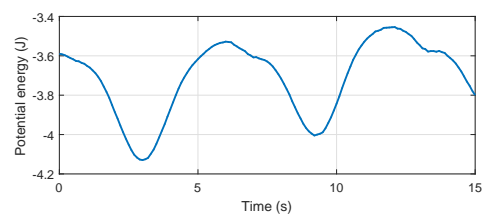

(a) Without potential energy minimization

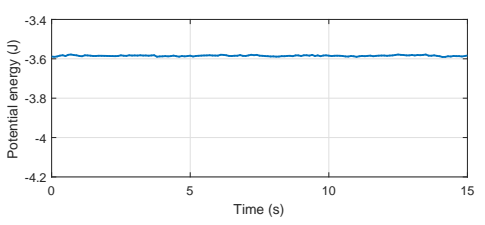

(b) With potential energy minimization

Figure 14: Potential energy $P$ in motion control process in $X-Y$ plane for the upper-limb exoskeleton system during the experiment. After potential energy minimization scheme is utilized, the oscillation magnitude of potential energy is evidently eliminated.

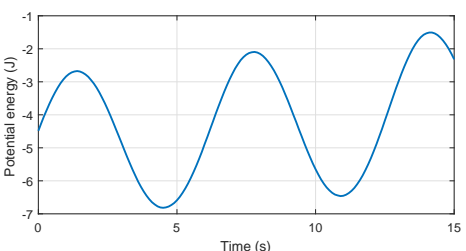

(a) Without potential energy minimization

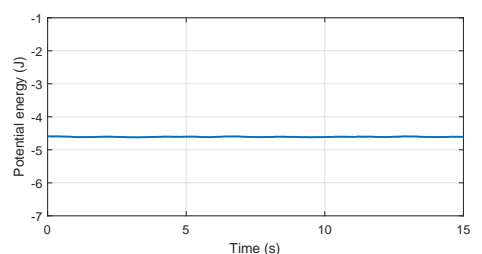

(b) With potential energy minimization

Figure 15: Potential energy $P$ in motion control process in $X-Z$ plane for the upper-limb exoskeleton system during the experiment. After potential energy minimization scheme is utilized, the oscillation magnitude of potential energy is evidently eliminated.

in $X-Y$ plane and $99.38 \%$ in $X-Z$ plane. These evaluations and comparisons on the potential energy variation demonstrate the efficiency of the resolution scheme based on proposed ZD-based potential energy minimization. Since the $X-Z$ plane is the vertical plane which is along the gravity acceleration, the potential energy modulation effect can be more evident by the proposed method as compared with the case in the $X-Y$ plane. This comparisons also indicate that the proposed method might be more efficient in weight-support applications.

\section{Conclusion}

In order to achieve accurate motion control of the upper-limb exoskeleton with minimal potential energy variation, we propose a new motion planning strategy with potential energy minimization involved. ZD method is utilized to guarantee the elegant convergence of solution and successfully obtain the inverse kinematics resolution accurately for real-time motion control of the upper-limb 
Table 3: PEVI values $(\mathrm{J} \cdot \mathrm{s})$ of the upper-limb exoskeleton system during motion control experiments.

\begin{tabular}{ccc}
\hline $\begin{array}{c}\text { Desired path plane } \\
\text { for motion control }\end{array}$ & $\begin{array}{c}\text { Without potential } \\
\text { energy minimization }\end{array}$ & $\begin{array}{c}\text { With potential } \\
\text { energy minimization }\end{array}$ \\
\hline$X-Y$ plane & 3.816 & $\mathbf{0 . 0 9 4}$ \\
$X-Z$ plane & 39.629 & $\mathbf{0 . 2 4 3}$ \\
\hline
\end{tabular}

exoskeleton. The upper-limb exoskeleton system takes advantage of the proposed scheme to achieve desired motion control goal and attempts to minimize its potential energy. Simulation results and experimental results demonstrate that the proposed motion planning scheme can make the upper-limb exoskeleton system track the desired motion path accurately. Moreover, such results demonstrate that, the potential energy variation range of the upper-limb exoskeleton can be significantly brought down by $99.34 \%$ in both $X-Y$ and $X-Z$ planes.

\section{Acknowledgement}

This work was supported by the National Key Research and Development Program of China under Grant No. 2017YFB1302304, in part by the National Natural Science Foundation of China under Grant 61603078, in part by the Hong Kong Research Grants Council Early Career Scheme under Grant 25214015, in part by the Departmental General Research Fund of The Hong Kong Polytechnic University under Grant G.UA7L.

\section{References}

[1] A. J. Young and D. P. Ferris, "State of the art and future directions for lower limb robotic exoskeletons," IEEE Transactions on Neural Systems and Rehabilitation Engineering, vol. 25, no. 2, pp. 171-182, Feb 2017.

[2] Z. Li, W. Ma, Z. Yin, and H. Guo, "Tracking control of time-varying knee exoskeleton disturbed by interaction torque," ISA Transactions, vol. 71, pp. $458-466,2017$. 
[3] S. Spada, L. Ghibaudo, S. Gilotta, L. Gastaldi, and M. P. Cavatorta, "Investigation into the applicability of a passive upper-limb exoskeleton in automotive industry," Procedia Manufacturing, vol. 11, pp. 1255 - 1262, 2017, 27th International Conference on Flexible Automation and Intelligent Manufacturing, FAIM2017, 27-30 June 2017, Modena, Italy.

[4] T. Proietti, V. Crocher, A. Roby-Brami, and N. Jarrass, "Upper-limb robotic exoskeletons for neurorehabilitation: A review on control strategies," IEEE Reviews in Biomedical Engineering, vol. 9, pp. 4-14, 2016.

[5] H. Kim, L. M. Miller, N. Byl, G. M. Abrams, and J. Rosen, "Redundancy resolution of the human arm and an upper limb exoskeleton," IEEE Transactions on Biomedical Engineering, vol. 59, no. 6, pp. 1770-1779, June 2012.

[6] B. Ugurlu, M. Nishimura, K. Hyodo, M. Kawanishi, and T. Narikiyo, "Proof of concept for robot-aided upper limb rehabilitation using disturbance observers," IEEE Transactions on Human-Machine Systems, vol. 45, no. 1, pp. 110-118, Feb 2015.

[7] Z. Li, D. Guiraud, and M. Hayashibe, "Inverse estimation of multiple muscle activations from joint moment with muscle synergy extraction," IEEE Journal of Biomedical and Health Informatics, vol. 19, no. 1, pp. 64-73, Jan 2015.

[8] Z. Li, H. Liu, Z. Yin, and K. Chen, "Muscle synergy alteration of humanduring walking with lower limb exoskeleton," Frontiers in Neuroscience, vol. 12 , no. 1050 , pp. 1-9, 2019.

[9] B. Siciliano and O. Khatib, Handbook of Robotics. Berlin, Heidelberg: Springer, 2008.

[10] Y. Nakamura, H. Hanafusa, and T. Yoshikawa, "Task-priority based redundancy control of robot manipulators," International Journal of Robotics Research, vol. 6, no. 2, pp. 3-15, 1987. 
[11] L. Siciliano and B. Sciavicco, "A solution algorithm to the inverse kinematic problem for redundant manipulators," IEEE Journal of Robotics and Automation, vol. 6, no. 2, pp. 3-15, 1987.

[12] Y. Zhang, J. Wang, and Y. Xia, "A dual neural network for redundancy resolution of kinematically redundant manipulators subject to joint limits and joint velocity limits," IEEE Transactions on Neural Networks, vol. 14, no. 3, pp. 658-667, May 2003.

[13] Y. S. Xia, Gang Feng, and Jun Wang, "A primal-dual neural network for online resolving constrained kinematic redundancy in robot motion control," IEEE Transactions on Systems, Man, and Cybernetics, Part B (Cybernetics), vol. 35, no. 1, pp. 54-64, Feb 2005.

[14] S. Li, Y. Zhang, and L. Jin, "Kinematic control of redundant manipulators using neural networks," IEEE Transactions on Neural Networks and Learning Systems, vol. 28, no. 10, pp. 2243-2254, Oct 2017.

[15] Z. Li, C. Li, S. Li, and X. Cao, "A fault-tolerant method for motion planning of industrial redundant manipulator," IEEE Transactions on Industrial Informatics, vol. 16, no. 12, 2019.

[16] B. Cai and Y. Zhang, "Different-level redundancy-resolution and its equivalent relationship analysis for robot manipulators using gradient-descent and zhang's neural-dynamic methods," IEEE Transactions on Industrial Electronics, vol. 59, no. 8, pp. 3146-3155, Aug 2012.

[17] L. Jin, Y. Zhang, S. Li, and Y. Zhang, "Modified znn for time-varying quadratic programming with inherent tolerance to noises and its application to kinematic redundancy resolution of robot manipulators," IEEE Transactions on Industrial Electronics, vol. 63, no. 11, pp. 6978-6988, Nov 2016.

[18] M. Plooij, U. Keller, B. Sterke, S. Komi, H. Vallery, and J. von Zitzewitz, "Design of rysen: An intrinsically safe and low-power three-dimensional 
overground body weight support," IEEE Robotics and Automation Letters, vol. 3, no. 3, pp. 2253-2260, July 2018.

[19] D. Wang, K. Lee, and J. Ji, "A passive gait-based weight-support lower extremity exoskeleton with compliant joints," IEEE Transactions on Robotics, vol. 32, no. 4, pp. 933-942, Aug 2016.

[20] G. Lv and R. D. Gregg, "Underactuated potential energy shaping with contact constraints: Application to a powered knee-ankle orthosis," IEEE Transactions on Control Systems Technology, vol. 26, no. 1, pp. 181-193, 2018.

[21] H. Yu, I. S. Choi, K.-L. Han, J. Y. Choi, G. Chung, and J. Suh, "Development of a upper-limb exoskeleton robot for refractory construction," Control Engineering Practice, vol. 72, pp. 104 - 113, 2018.

[22] D. Guo and Y. Zhang, "Zhang neural network for online solution of timevarying linear matrix inequality aided with an equality conversion," IEEE Transactions on Neural Networks and Learning Systems, vol. 25, no. 2, pp. 370-382, Feb 2014.

[23] Yunong Zhang and S. S. Ge, "Design and analysis of a general recurrent neural network model for time-varying matrix inversion," IEEE Transactions on Neural Networks, vol. 16, no. 6, pp. 1477-1490, Nov 2005.

[24] Z. Li and Y. Zhang, "Improved zhang neural network model and its solution of time-varying generalized linear matrix equations," Expert Systems with Applications, vol. 37, no. 10, pp. 7213 - 7218, 2010. 